\title{
Shear-wave splitting analysis of later phases in southwest Japan -A lineament structure detector inside the crust-
}

\author{
Takashi Iidaka \\ Earthquake Research Institute, University of Tokyo, Yayoi 1-1-1, Bunkyo, Tokyo 113-0032, Japan
}

(Received March 28, 2003; Revised May 28, 2003; Accepted May 31, 2003)

\begin{abstract}
We attempt to detect the lineament structure inside the crust using a shear-wave splitting analysis. A seismicity map suggests several lineaments in southwest Japan. No clear active fault was found to support the lineaments. We researched shear-wave splitting in southwest Japan using two later phases: PpPms and PpSms. The PpPms is a later $S$-phase, which is reflected at the surface as a $P$ wave, dives again, and reflected and converted from $P$ to $S$ at the Moho boundary. The PpSms is a later arriving $S$-phase, which is converted from $P$ at the Earth's surface, and is reflected at the Moho discontinuity before arriving at a seismic station. The PpPms and PpSms phases are used to detect lineament structures inside the crust. The observed polarization directions obtained are ENE-WSW, NE-SW, and N-S with a lateral variation. The directions are not consistent with that of maximum principal stress in the area. The results of shear-wave splitting in southwest Japan could not be explained by crack-induced anisotropy caused by maximum principal stress. The direction NE-SW is consistent with that of the geological lineament structure in this area. The shear-wave splitting is probably caused by the lineament structure inside the crust.
\end{abstract}

Key words: Shear-wave splitting, southwest Japan, later phase, anisotropy.

\section{Introduction}

Shear-wave splitting analyses have provided us with a new source of information to study the seismic structure and mantle dynamics. The causes of anisotropy are considered to be the preferred orientation of anisotropic crystal and crack-induced heterogeneous structure (e.g., Crampin, 1981). Mapping the shear-wave splitting data using crustal earthquakes in Japan showed that the polarization direction was almost consistent with the direction of maximum principal stress (Kaneshima, 1990). Shear-wave splitting in the upper crust was considered to be caused by cracks, which was opened by maximum principal stress. In continental areas, however, shear-wave splitting was directly related to internal deformation caused by past tectonic processes such as orogeny (e.g., Silver and Chan, 1991; Park and Levin, 2002). The lineament structure inside the crust could be a cause of the shear-wave splitting.

In the southwest Japan region, a seismicity map shows clear lineaments of the location of micro-earthquakes (e.g., Nakamura et al., 1997). The most remarkable lineament of seismicity is almost parallel to the northern coastline in the western part of Japan. The directions of the lineaments are E-W and NE-SW at the east and west parts of western Japan, respectively. However, no clear active fault is found along the lineaments. The existence of a lineament structure inside the crust is suspected. In the southwest Japan region, the direction of maximum principal stress is obtained to be NWSE (e.g., Ando, 1979; Tsukahara and Kobayashi, 1991). If the cause of shear-wave splitting in the crust is the opening

Copy right(C) The Society of Geomagnetism and Earth, Planetary and Space Sciences (SGEPSS); The Seismological Society of Japan; The Volcanological Society of Japan; The Geodetic Society of Japan; The Japanese Society for Planetary Sciences. of cracks by stress, the observed shear-wave splitting data should be polarized to NW-SE. However, if the polarization direction is not NW-SE other causes have to be considered. The lineament structure could be a cause of anisotropy. Because shear-wave splitting was directly related to internal deformation caused by past tectonic processes such as orogeny (e.g., Silver and Chan, 1991; Park and Levin, 2002).

A weak point of the shear-wave splitting analysis is low resolution in the depth direction, because the observed anisotropy is the accumulated anisotropy where the shearwave traveled. Iidaka and Niu (2001) attempt to use converted waves to obtain the anisotropy of each layer (i.e., crust, upper mantle, and lower mantle). The PpSms (the notation is defined by Bath and Stefansson, 1966) is a laterarriving $S$-phase, which is converted from $P$ at the earth's surface, and is reflected at the Moho discontinuity before arriving at a seismic station. Iidaka and Niu (2001) first used the PpSms phase for the shear-wave splitting analysis. PpPms is a later $S$-phase, which is reflected at the surface as a $P$ wave, dives again, and is reflected and converted from $P$ to $S$ at the Moho boundary. PpPms and PpSms waves were proved to be good waves to detect whole crustal anisotropy. We try to detect crustal anisotropy using the later phases of PpPms and PpSms.

\section{Data}

We used J-array seismic stations at latitudes $34^{\circ} \mathrm{N}-36^{\circ} \mathrm{N}$, longitudes $131^{\circ} \mathrm{E}-134.5^{\circ} \mathrm{E}$ (Fig. 1). J-array stations cover the whole of the Japan area with short-period seismometers. Most of the seismic stations use a seismometer with a natural frequency of $1 \mathrm{~Hz}$. The sampling frequency of the data is 20 $\mathrm{Hz}$.

The large earthquakes are used with a period of January 1, 


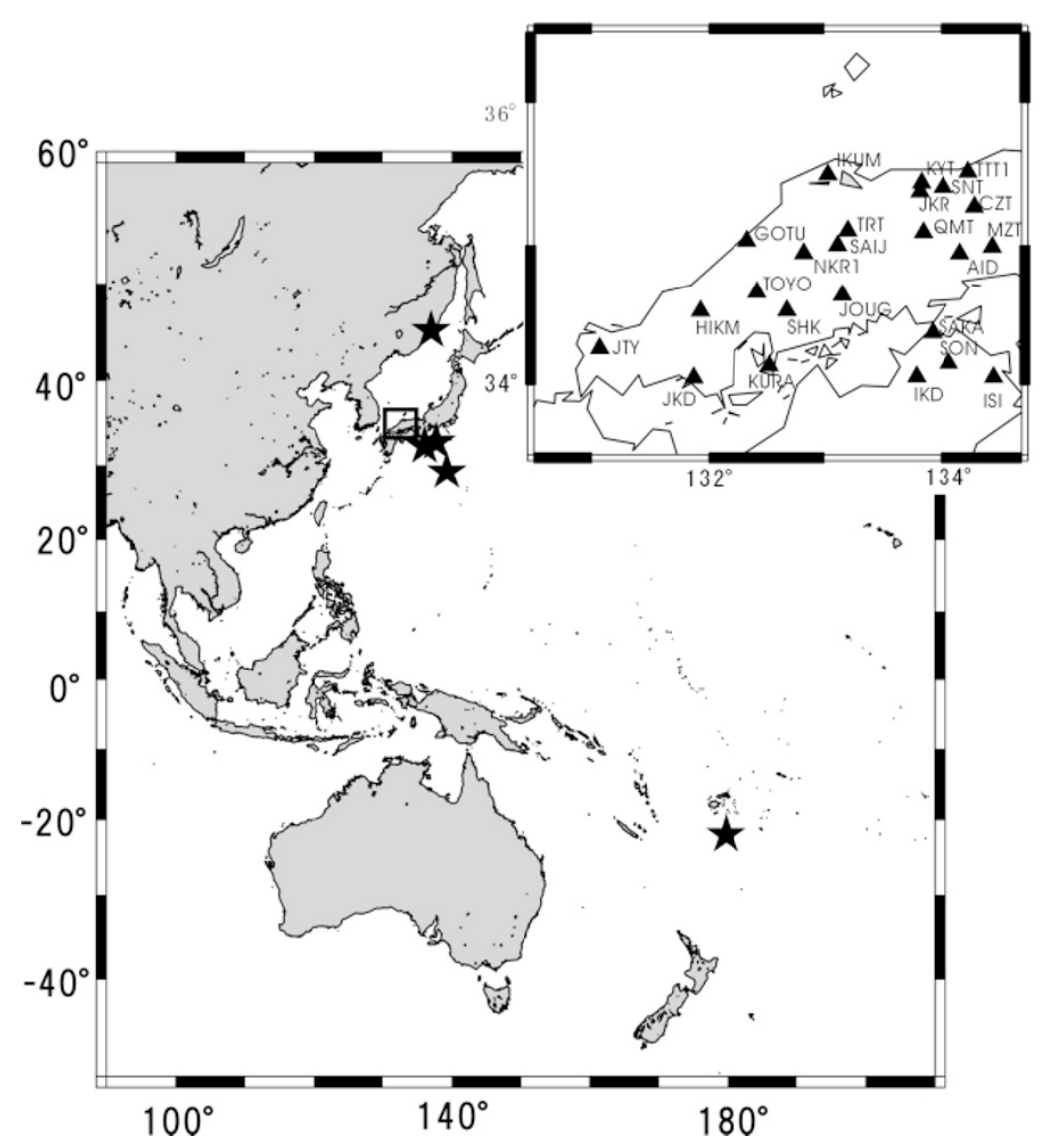

Fig. 1. Map of earthquakes (stars) used in this study. Seismic stations are shown by triangles in the inset.

2001-October 31, 2002. The earthquakes that clear PpPms and PpSms waves observed are used for the analysis (Fig. 1) (Table 1). Deep earthquakes with a depth greater than $200 \mathrm{~km}$ are used, because the waveforms of shallow earthquakes are contaminated by reflected and converted phases near the source area.

\section{Method}

\subsection{PpPms and PpSms phases}

PpPms and PpSms are reverberated phases between the surface and the Moho discontinuity (Fig. 2). PpPms and PpSms make one-way and two-way traverses through the crust, respectively, along a near vertical path as an $S$ wave. However, we have to take care with the PpPms and PpSms phases used for the analysis (e.g., Niu and James, 2002). The arrival time of the PpPms is the same as those of PsPmp and PpSmp (2p1s) (Fig. 2). The three phases consist of two-way paths with $S$-wave velocity and one-way path with $P$-wave velocity. On the other hand, the arrival time of PpSms is the same as those of PsPms and PsSmp (1p2s), which consists of one-way path of $P$-wave and two-way paths with $S$-wave (Fig. 2).

The later arrival of $2 \mathrm{p} 1 \mathrm{~s}$ consists of three phases, PpPms, PsPmp, and PpSmp, with almost the same arrival times. The phases of PsPmp and PpSmp are $P$-wave. But, the phase of PpPms is an $S$-wave. The incident angles of the three phases are almost vertical. The amplitude of the two phases (PsPmp and PpSmp) is considered to be small on the horizontal components. The PpPms is not contaminated by other phases on the horizontal components.

The later arrival of $1 \mathrm{p} 2 \mathrm{~s}$ consists of three later phases: PpSms, PsPms, and PsSmp. Both of the later PpSms and $P s P m s$ phases are $S$-waves. The amplitude of the two phases on the horizontal components has to be estimated, because the two phases arrive at a seismic station at almost the same time as an $S$-wave. The PpSms includes crustal anisotropy of the two-way paths and the PsPms includes one-way path crustal anisotropy. A ray-tracing method, SEIS83, is used to calculate the amplitudes of the two phases on the horizontal components (Cerveny and Psencik, 1983). The amplitude for the radial component of the PpSms phase is 20-50 times larger than that of PpSms in the case of incident angles of 20-30 deg. The amplitude of PpSms on the horizontal component is much larger than that of other phases at the arrival time of $1 \mathrm{p} 2 \mathrm{~s}$.

PpPms and PpSms have the following advantages for analyzing shear-wave splitting. (a) PpPms and PpSms are free from the effects of mantle anisotropy, because it does not travel in the mantle as a shear-wave. (b) The amplitudes of PpPms and PpSms are expected to be large in the synthetic calculation using the Thomson-Haskell method (Haskell, 1962) and ray-tracing method (Cerveny and Psencik, 1983). (c) PpPms and PpSms phases are isolated from the $P$-coda wave. The arrival times of $P p P m s$ and $P p S m s$ are much later than $P$ arrivals, because PpPms and PpSms make two-way traverses through the crust.

The PpSms-phase was first applied for the analysis of crustal anisotropy by Iidaka and Niu (2001). The PpPms 


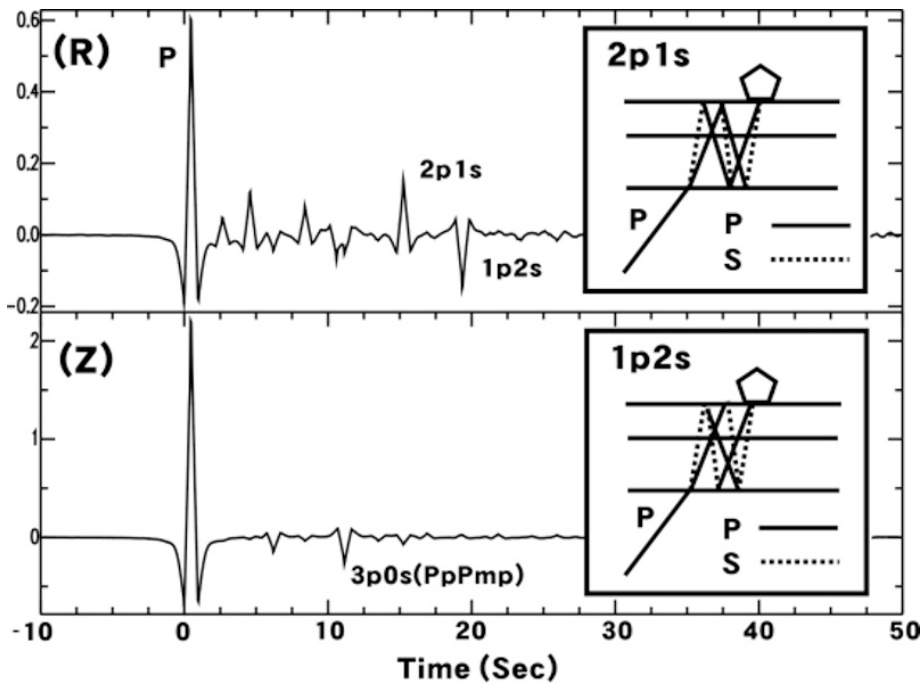

Fig. 2. Schematic ray paths of later phases are shown in the inset. Theoretical vertical $(\mathrm{Z})$ and radial (R) component seismograms are calculated by Thomson-Haskell method. The crustal structure model of Yoshii et al. (1974) is used. In the mantle, the iasp91 seismic velocity model is used. The later phase 2p1s (two $P$-wave paths and one $S$-wave path in the crust) consists of $P p P m s, P p S m p$, and $P s P m p$. The later phase 1 p2s (one $P$-wave path and two $S$-wave paths in crust) consists of PpSms, PsPms, and PsPmp.

Table 1. List of Earthquakes

\begin{tabular}{ccccc}
\hline Origin Time & Lat. $(\mathrm{deg})$ & Long. $(\mathrm{deg})$ & Dep. $(\mathrm{km})$ & $\mathrm{M}$ \\
\hline $2001042313: 55: 27.81$ & 33.02 & 136.61 & 417 & 5.4 \\
20011111 18:12:46.57 & 33.28 & 137.39 & 362 & 5.0 \\
$2002020121: 55: 20.99$ & 45.46 & 136.72 & 355 & 6.2 \\
$2002063021: 29: 36.30$ & -22.20 & 179.25 & 620 & 6.5 \\
$2002080223: 11: 39.13$ & 29.28 & 138.97 & 426 & 6.3 \\
\hline
\end{tabular}

and PpSms phases had the following characteristics. (1) Its amplitudes are largest on the radial component, because both phases arrive at the stations as an $S$-wave. (2) The PpPms- $P$ time and $P p S m s-P$ time are not sensitive to epicentral distance. (3) The PpPms-P time and PpSms-P time are also not sensitive to the depth and the location of the earthquakes.

In southwestern Japan, a crustal structure was obtained by a seismic experiment with explosive sources (Yoshii et al., 1974). Theoretical $P$ and $S V$ seismograms are calculated using the Thomson-Haskell method (Haskell, 1962) and raytracing method (Cerveny and Psencik, 1983) with the crustal structure model. Theoretical calculations show that the amplitudes of PpPms and PpSms phases are larger than other later arrivals. The PpPms and PpSms phases are expected to arrive about $15 \mathrm{sec}$ and $20 \mathrm{sec}$ after the direct $P$-phase, respectively. A clear isolated-phase, suggesting the above characteristics, within the expected time-windows is picked up as PpPms and PpSms phases (Fig. 3).

\subsection{Shear-wave splitting detection}

Shear-wave splitting is usually expressed by two parameters, which are time-lag $\tau$ (in seconds), time between the fast and slow components of the split shear wave, and fast polarization azimuth $\phi$ (in degrees). We investigate shear-wave splitting using techniques employed by Fukao (1984). After the seismic signals of PpPms and PpSms waves are pickedup from individual seismograms, the following process is then used to determine the shear-wave splitting. The ob-

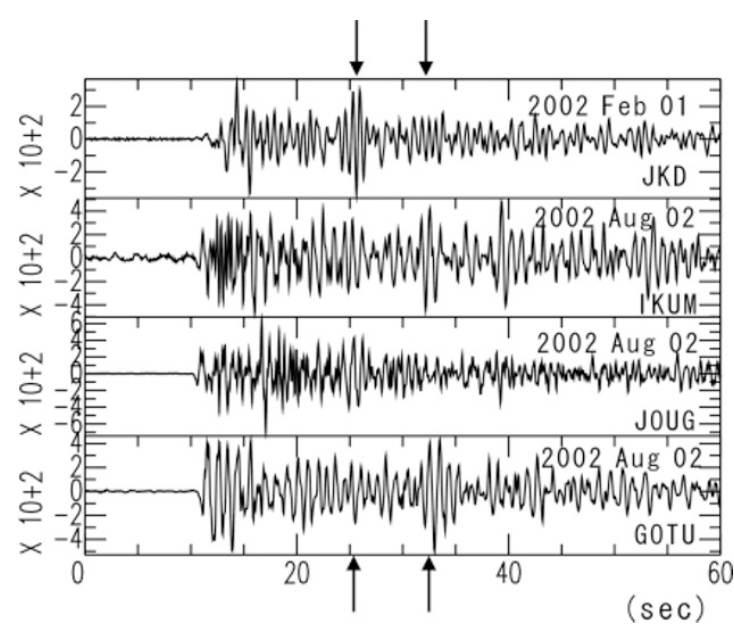

Fig. 3. Examples of waveforms of the later phases of 2p1s (left arrow) and of $1 \mathrm{p} 2 \mathrm{~s}$ (right arrow) on the radial component at several seismic stations. The name of the seismic station is shown at the bottom right corner.

served later phases are first divided into two orthogonal components. The two waveforms on the two orthogonal components are then rotated with a spacing of 1 degree azimuthal intervals. The cross-correlation of the two waveforms is calculated with one sample of time interval at each 1 degree azimuthal interval. The time lag $\tau$ (in seconds) and the fast polarization azimuth $\phi$ (in degree) are defined to be the val- 
a)

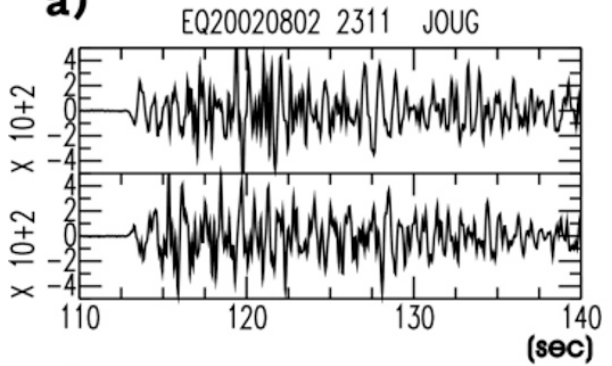

b)

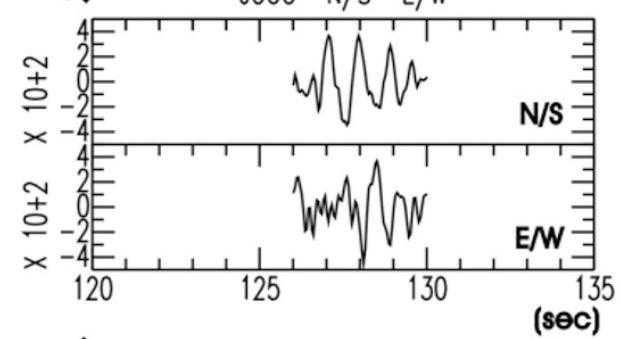

c) JOUG Rotated Time-shifted

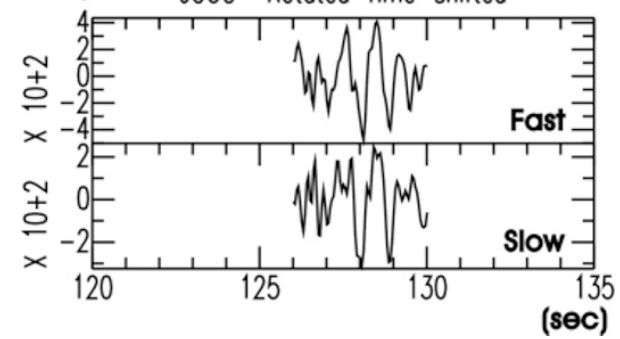

d)

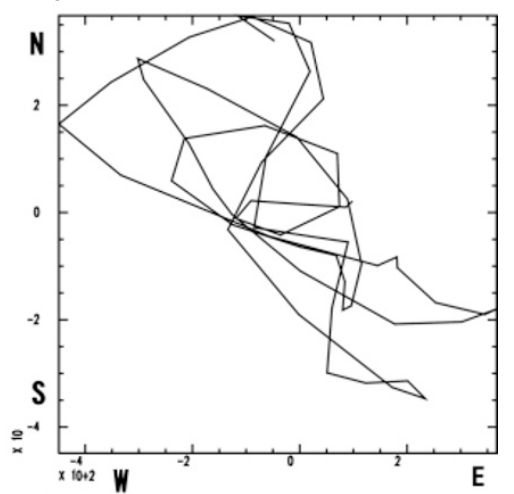

e)

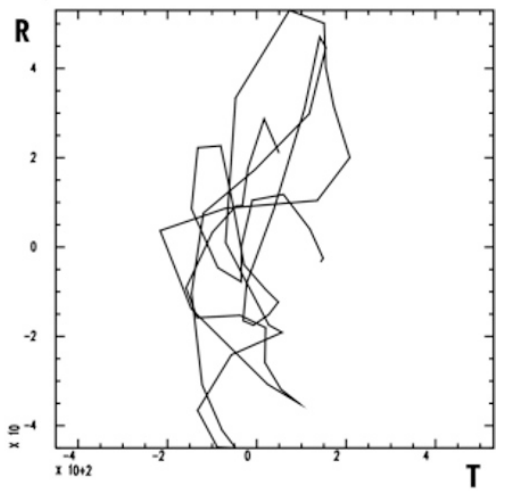

Fig. 4. Examples of seismograms of 2p1s wave (a). Original N-S, E-W components (b) and the two components that have a maximum cross-correlation (c). The particle motions of original and anisotropy-corrected waveforms are shown in (d) and (e), respectively.

ues that yielded the maximum correlation (Fig. 4).

The values obtained of azimuth and time lag are checked as follows. Anisotropy-corrected seismograms are obtained by a clockwise rotation $\phi$ and a relative time shift $\tau$. Appropriate rotation and time shift would show a linear orbit, which is expected to be in the radial direction, in the resultant particle motion diagram. The data indicating linear orbits obtained from anisotropy-corrected seismograms are used to exclude artificial results in which waveforms were distorted by noise.

\section{Results and Discussion}

The observed data suggest polarization directions of ENEWSW, NE-SW, and N-S with a lateral variation in the research area (Fig 5). The time-lag data $\tau$ vary from $0 \mathrm{sec}$ to $1.45 \mathrm{sec}$. A large lateral variation is found in the $\tau$-values. However, the polarization direction suggests a similar direction with a lateral variation.

Several models are considered as causes of the anisotropic structure. These are 1) preferred orientation of anisotropic crystal (e.g., Crampin, 1981), 2) heterogeneous structure with melt-filled cracks (e.g., Iidaka and Obara, 1995; Hiramatsu et al., 1998), 3) crack-induced heterogeneous structure (e.g., Crampin, 1981; Kaneshima, 1990), 4) heterogeneous structure caused by the internal deformation from a past tectonic process (e.g., Silver and Chan, 1991).

The preferred orientation of the anisotropic crystal model is usually used to explain the observed mantle anisotropy, because olivine crystal, which has a large anisotropy, is the most popular component of the mantle. In the mantle, a model that has a heterogeneous structure with melt filled cracks has been presented as the cause of anisotropy (e.g., Iidaka and Obara, 1995; Hiramatsu et al., 1998). If the meltfilled cracks are the cause of anisotropy, a large anisotropy should be observed volcanic area. In southwest Japan, several volcanoes are located along the coastline at the northwestern part of the research area. The observed splitting values in the area are not large compared to other regions. This model cannot explain the observed data.

The map of the anisotropy in Japan shows that the polarization direction of crustal anisotropy is very similar to the direction of maximum principal stress (Kaneshima, 1990). The cause of anisotropy in the crust is considered to be crackinduced heterogeneous structure. In southwest Japan, the direction of maximum principal stress obtained is NW-SE (e.g. Ando, 1979; Tsukahara and Kobayashi, 1991). If the cause of the anisotropy is opening-cracks in the upper crust, the polarization direction should be NW-SE. The direction of the observed data is not NW-SE. It is clear that the cause of the observed anisotropy cannot be explained by opening-cracks in the crust. Other causes have to be considered.

Next, a possible model of the heterogeneous structure caused by internal deformation is considered. Seismic anisotropy at continents was researched from shear-wave splitting records (Silver and Chan, 1991; Silver, 1996). The shear-wave splitting was directly related to internal defor- 


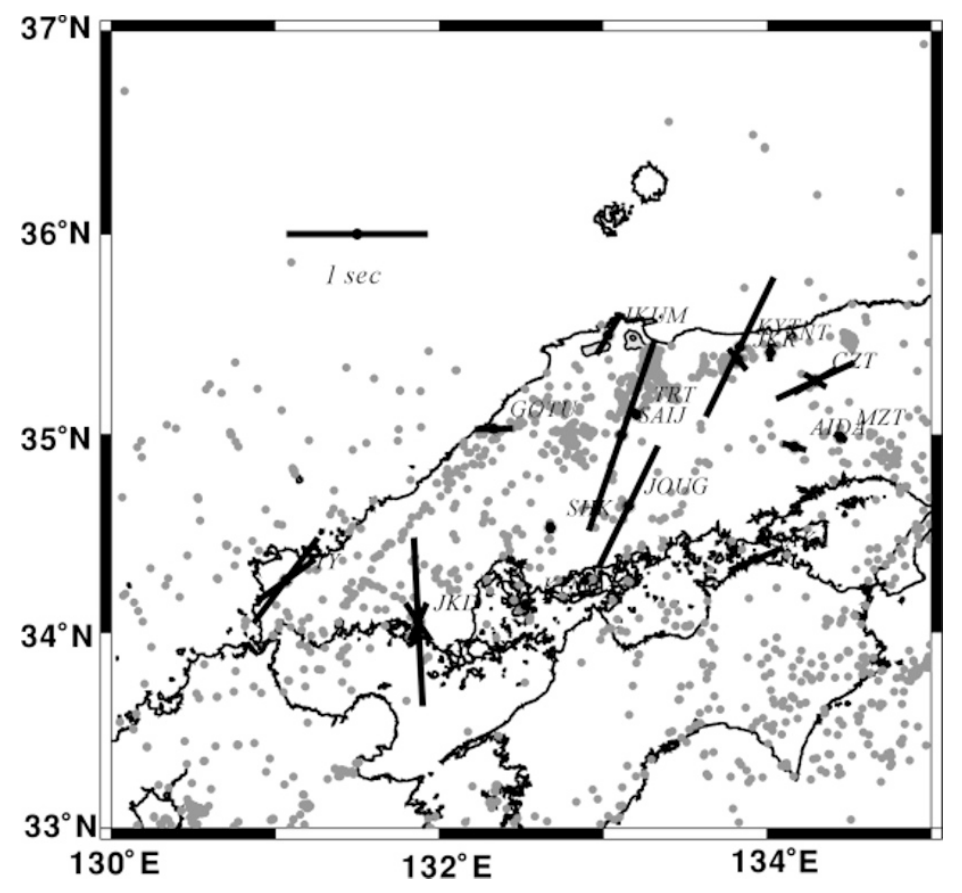

Fig. 5. Results of observed shear-wave splitting in southwest Japan. Polarized azimuth of maximum-velocity phase and time delay between maximum and minimum velocity phases are shown by the direction and the length of the bar, respectively. The circles are hypocenters which were determined by Japan Meteorological Agency (JMA). The earthquakes, which occurred in a period of Jan. 1, 2001-Sep. 30, 2002, are used. The depths of the earthquakes are shallower than $30 \mathrm{~km}$. The hypocenters data are selected using a tool, which was made by Tsuruoka (1998).

mation by past tectonic processes such as orogeny. In the southwestern Japan region, the strike of geological segmentation is roughly ENE-WSW from geological studies (e.g., Isozaki and Maruyama, 1991). Paleomagnetic studies suggest a clockwise rotation of southwest Japan associated with the opening of Japan Sea (e.g., Otofuji and Matsuda, 1983). A lineament structure in the NE-SW direction is expected because the direction is roughly normal to the direction of the opening of Japan Sea. Petrological studies also suggest a block structure with a lineation in the ENE-WSW direction existed in southwest Japan (Iwamori, 1991). Much evidence for the existence of a lineament structure in the directions NE-SW and ENE-WSW has been presented in this area. The deformation process might make some lineament structures. The "fossil" structure could be a cause of the shear-wave splitting observed in this study. The shear-wave splitting might be caused by the lineament structure inside the crust.

The observed data suggest large lateral variations of $\tau$ values. If the deformation process makes a lineament structure in this area, a large lateral variation of spatial density of lineaments is expected because the crust is not uniform, resulting in large lateral variations of anisotropy values. The observed $\tau$-values are much larger than that of crustal anisotropy caused by opening of cracks, which is the maximum $\tau$-value of $0.2 \mathrm{sec}$. The large anisotropy is also supported by the fact that the observed data cannot be explained by the crack-induced heterogeneous structure.

Kaneshima (1990) showed shear-wave splitting values for the whole Japan area. However, few observed data were obtained for this research area. The observed data were located in the east-most part of the research area in this study. He suggested a polarization direction of ESE-SNS at the east-most part of the research area. This study suggests ESESNS at the seismic stations of AIDA and MZT, and ENEWSW at the seismic station of CZT. The direction is not so different from his results.

The maximum principal stress direction is NW-SE in this area. Shear-wave splitting caused by the opening of cracks by stress have to be observed. However, the magnitude of the anisotropy is small compared to the observed splitting $\tau$ values. The anisotropy caused by stress might be covered by the large anisotropy caused by internal deformation due to the tectonic process.

Crustal earthquakes have been used for the shearwave splitting analysis to detect crustal anisotropy (e.g., Kaneshima, 1990). The dominant frequency of the crustal earthquakes is about $10 \mathrm{~Hz}$. On the other hand, the dominant frequency of the later phases is around $1 \mathrm{~Hz}$. These frequency ranges are different. Recently, a difference in the magnitude of anisotropy in different frequency bands was reported (e.g., Marson-Pidgeon and Savage, 1997). When evaluating the magnitude of anisotropy using different phases one must consider the effects on observed anisotropic parameters. A more detail analysis is required for the evauation of the magnitude of anisotropy.

In this area, observation of temporally seismic array is conducted by a joint project of universities (Japanese University Group of the Joint Seismic Observations at the Southwestern Japan, 2002). The array consists of 40 seismic stations with satellite telemetry systems. This array will be operated from 2002 to 2004 . In this study, a lack of data prevents us for knowing the cause and magnitude of anisotropy. However, the data of the array will reveal the cause and the magnitude of anisotropy, and suggest some lineament structures in southwest Japan. 


\section{Conclusions}

Crustal anisotropy was detected by analyzing PpPms and PpSms phases. PpPms and PpSms are reverberated phases between the surface and the Moho discontinuity. The polarization directions of the observed anisotropy are ENE-WSW, NE-SW, and N-S with regional variations. The direction is different from that of the maximum principal stress. The cause of anisotropy could not be explained by the opening of cracks by stress. The NE-SW direction is consistent with the direction of geological lineation. The existence of a lineament structure in southwest Japan has been suggested by geological and petrological studies. A lack of the data prevents us from revealing the cause of anisotropy. Shear-wave splitting is probably caused by the lineament structure. Seismic observations with a highly dense network will make clarify the cause and the magnitude of anisotropy.

Acknowledgments. I thank Prof. Iwamori for helpful discussions. Seismic Analysis Code (SAC) was used in our calculations. I thank Prof. Niu for providing computer programs. I also thank for anonymous reviewers for their comments and suggestions. The hypocenter location data, which were provided by Japan Meteorological Agency, are used.

\section{References}

Ando, M., The stress field of the Japan Islands in the last 0.5 million years, Earth Mon. Symp., 7, 541-546, 1979.

Bath, M. and R. Stefansson, S-P conversion at the base of the crust, Annali di Geofisica, 19, 119-130, 1966.

Cerveny, V. and I. Psencik, Program Package SEIS83, 1983.

Crampin, S., A review of wave motion in anisotropic and cracked elasticmedium, Wave Motion, 3, 343-391, 1981.

Fukao, Y., Evidence from core-reflected shear waves anisotropy in the Earth's mantle, Nature, 309, 695-698, 1984.

Haskell, N. A., Crustal reflections of the plane P and SV waves, J. Geophys. Res., 67, 4751-4767, 1962.

Hiramatsu, Y., M. Ando, T. Tsukuda, and T. Ooida, Three-dimensional image of the anisotropic bodies beneath central Honshu, Japan, Geophys. J. Int., 135, 801-816, 1998.
Iidaka, T. and F. Niu, Mantle and crustal anisotropy in the eastern China region inferred from waveform splitting of SKS and PpSms, Earth Planets Space, 53, 159-168, 2001.

Iidaka, T. and K. Obara, Shear-wave polarization anisotropy in the mantle wedge above the subducting Pacific plate, Tectonophys., 249, 53-68, 1995.

Isozaki, Y. and S. Maruyama, Studies on orogeny based on plate tectonics in Japan and new geotectonic subdivision of the Japanese Islands, Journal of Geography, 100, 697-761, 1991.

Iwamori, H., Zonal structure of Cenozoic basalts related to mantle upwelling in southwest Japan, J. Geophys. Res., 96, 6157-6170, 1991.

Japanese University Group of the Joint Seismic Observations at the Southwestern Japan, The joint seismic observations at the southwestern Japan, Abst. Seismo. Soc. Japan Fall Meeting, P004, 2002.

Kaneshima, S., Original of crustal anisotropy: Shear wave splitting studies in Japan, J. Geophys. Res., 95, 11121-11133, 1990.

Marson-Pidgeon, K. and M. Savage, Frequency-dependent anisotropy in Wellington, New Zealand, Geophys. Res. Lett., 24, 3297-3300, 1997.

Nakamura, M., H. Watanabe, T. Konomi, S. Kimura, and K. Miura, Characteristic activities of subcrustal earthquakes along the outer zone of southwestern Japan, Annuals of Disas. Prev. Res. Inst., Kyoto Univ., 40, 1-20, 1997.

Niu, F. and D. James, Fine structure of the lowermost crust beneath the Kaapvaal craton and its implications for crustal formation and evolution, Earth and Planet. Sci. Lett., 200, 121-130, 2002.

Otofuji, Y. and T. Matsuda, Paleomagnetic evidence for the clockwise rotation of Southwest Japan, Earth Planet. Sci. Lett., 62, 349-359, 1983.

Park, J. and V. Levin, Seismic anisotropy: tracing plate dynamics in the mantle, Science, 296, 485-489, 2002.

Silver, P. G., Seismic anisotropy beneath the continents: Probing the depths of geology, Annu. Rev. Earth Planet. Sci., 24, 385-432, 1996.

Silver, P. G. and W. Chan, Shear wave splitting and subcontinental mantle deformation, J. Geophys. Res., 96, 16429-16454, 1991.

Tsukahara, H. and Y. Kobayashi, Crustal stress in the central and western parts of Honshu, Japan, Zisin, 44, 221-231, 1991.

Tsuruoka, H., Development of earthquake information retrieval and analysis system on WWW, I.P.S.J. SIG Notes, 98, 65-70, 1998.

Yoshii, T., Y. Sasaki, T. Tada, H. Okada, S. Asano, I. Muramatsu, M Hashizume, and T. Moriya, The third Kurayoshi explosion and the crustal structure in the western part of Japan, J. Phys. Earth, 22, 109-121, 1974

T. Iidaka (e-mail: iidaka@eri.u-tokyo.ac.jp) 